\title{
TRALI in the perioperative period
}

This article was published in the following Dove Press journal:

International Journal of Clinical Transfusion Medicine

3 July 2014

Number of times this article has been viewed

\section{Mohamed Adnane Berdai \\ Smael Labib \\ Mustapha Harandou \\ Child and Mother Intensive Care Unit, University Hospital Hassan II, Fes, Morocco}

Correspondence: Mohamed Adnane Berdai Child and Mother Intensive Care Unit, University Hospital Hassan II, Sidi Hrazem Avenue, 30000, Fes, Morocco

Tel +212655260752

Email adnane.berdai@yahoo.fr
Background: Transfusion-related acute lung injury (TRALI) is one of the leading causes of transfusion-related morbidity and mortality. Although it is a serious complication of blood transfusion, it is still underestimated because of underrecognition and misdiagnosis, which can lead to inappropriate management.

Case report: We report the case of a 34-year-old female, who presented with hemorrhagic shock during cesarean section and was transfused with packed red blood cells and fresh frozen plasma. Three hours after the end of transfusion, while still intubated, the patient developed acute respiratory failure, with diffuse crackles at auscultation and bilateral lung infiltrations on chest radiography. The ratio of the arterial partial pressure of $\mathrm{O}_{2}$ and the fraction of inspired $\mathrm{O}_{2}$ $\left(\mathrm{PaO}_{2} / \mathrm{FiO}_{2}\right)$ was 51 . The diagnosis of TRALI was made after excluding other possible causes of acute lung injury. She was managed symptomatically with ventilatory support and vasopressors. The patient later died secondary to multiple organ failure.

Conclusion: The diagnosis of TRALI relies on the exclusion of other causes of acute pulmonary edema following transfusion. All plasma-containing blood products have been involved in the genesis of this syndrome. This is a relatively common and serious adverse transfusion reaction that requires prompt diagnosis and management. Challenges are ahead as preventive strategies have reduced but not eliminated the onset of TRALI.

Keywords: acute lung injury, transfusion related acute lung injury, anti-human leukocyte antigen antibody, pulmonary edema, transfusion accident

\section{Introduction}

Transfusion-related acute lung injury (TRALI) is a clinical syndrome that presents as acute hypoxemia and noncardiogenic pulmonary edema during or after blood transfusion. ${ }^{1}$ In the United States, TRALI is among the most common causes of transfusion reactions, being considered as the leading cause of transfusion-related mortality. In 2005-2009, 48\% of the confirmed transfusion-related deaths in the USA that were reported to the Food and Drug Administration (FDA) were secondary to TRALI. ${ }^{2}$ However, this syndrome is still underdiagnosed and underreported in developing countries because of the lack of recognition.

We report the case of a pregnant woman who developed TRALI after transfusion during cesarean section, and we discuss the clinical and therapeutic aspects of this syndrome.

\section{Case report}

A 34-year-old woman was hospitalized in the obstetric ward for placenta previa totalis. She had a history of cesarean section under general anesthesia 14 years prior, with an 
uneventful perioperative period. She had no previous history of any medical illness or comorbidities. During hospitalization, the preanesthetic evaluation did not reveal any abnormal findings, except for a hemoglobin level of $9.3 \mathrm{~g} / \mathrm{dL}$. Her blood group was $\mathrm{O}$ positive, and she was transfused with two units of packed red blood cells (PRBCs); otherwise, the coagulation profile was normal. Three days later, she underwent cesarean section for placenta previa totalis. On admission in the operating room, the patient had a regular pulse with heart rate (HR) of $98 \mathrm{bpm}$, blood pressure of 120/80 $\mathrm{mmHg}$, and pulse oximetry saturation $\left(\mathrm{SpO}_{2}\right)$ of $99 \%$. Spinal anesthesia was planned for the surgery. The subarachnoid block was performed with 12 $\mathrm{mg}(2.4 \mathrm{~mL})$ of $0.5 \%$ bupivacaine and $25 \mu \mathrm{g}$ of fentanyl, which were injected through a 27 gauge needle at the midline of the L3-L4 interspinous space after cerebrospinal fluid aspiration, while the patient was in a sitting position. Approximately 5 minutes after incision, the fetus was delivered. Intraoperative findings revealed placenta accreta, and the placental delivery was impossible, so the decision to perform total hysterectomy was taken. Meanwhile, the patient presented continuous bleeding, estimated at $1.1 \mathrm{~L}$. It was decided to electively intubate and ventilate, in view of the continued bleeding, hemodynamic instability, and prolonged surgery.

Following 3 minutes of preoxygenation, the patient was induced in rapid sequence with etomidate, $0.3 \mathrm{mg} / \mathrm{kg}$, and rocuronium, $0.9 \mathrm{mg} / \mathrm{kg}$. Cricoid pressure was applied from loss of consciousness until confirmation of tracheal cuffed tube positioning. We then administered fentanyl at $3 \mu \mathrm{g} / \mathrm{kg}$. An internal jugular vein catheter and a radial arterial line were inserted. The blood pressure was maintained with a noradrenaline infusion at $0.5 \mu \mathrm{g} / \mathrm{kg} / \mathrm{min}$. The anesthesia was maintained with sevoflurane, fentanyl, and rocuronium. During surgery, blood loss was estimated at $2 \mathrm{~L}$, and the urine output was $200 \mathrm{~mL}$, the total volume of infused crystalloid was $1 \mathrm{~L}$, associated with the transfusion of four units of PRBC and four units of fresh frozen plasma (FFP). The patient also received $20 \mathrm{~mL}$ of calcium gluconate. The hysterectomy was performed and good hemostasis was achieved. The patient was shifted to the intensive care unit (ICU) and continued on ventilator support, sedated with midazolam, $0.1 \mathrm{mg} / \mathrm{kg} / \mathrm{h}$, and fentanyl, $1 \mu \mathrm{g} / \mathrm{kg} / \mathrm{h}$. At the ICU, the patient required only a low dose of noradrenaline, at $0.1 \mu \mathrm{g} / \mathrm{kg} / \mathrm{min}$. The urine output was $2 \mathrm{~mL} / \mathrm{kg} / \mathrm{h}$. Three hours after the end of transfusion, she developed tachycardia (HR at $130 \mathrm{bpm}$, regular), with fever of $105^{\circ} \mathrm{F}$ and sweating, and production of clear frothy fluid secretions. The lung auscultation revealed diffuse crackles; at that time, the $\mathrm{SpO}_{2}$ dropped to $60 \%$ despite an increase in the fraction of inspired oxygen $\left(\mathrm{FiO}_{2}\right)$ to
$100 \%$. Chest radiography revealed bilateral lung infiltrations (Figure 1). Arterial blood gas analysis showed a pH of 7.23, a partial pressure of $\mathrm{CO}_{2}$ in the blood $\left(\mathrm{PaCO}_{2}\right)$ of $38 \mathrm{mmHg}$, an arterial partial pressure of $\mathrm{O}_{2}\left(\mathrm{PaO}_{2}\right)$ of $51 \mathrm{mmHg}$, and an arterial oxygen saturation $\left(\mathrm{SaO}_{2}\right)$ of $85 \%$ despite her mechanical ventilatory support at $\mathrm{FiO}_{2}$ of $100 \%$ and positive end-expiratory pressure (PEEP) at $10-15 \mathrm{cmH}_{2} \mathrm{O}$.

Fluid overload was ruled out by a normal central venous pressure (CVP) of $5 \mathrm{cmH}_{2} \mathrm{O}$ and normal echocardiography that revealed no abnormalities of the heart valves, no hypokinesia of the myocardium, and an ejection fraction of $65 \%$. We also noted the absence of typical clinical features of anaphylactic reaction or toxic shock, such as bronchospasm, rashes, and urticaria. There was no evidence of aspiration pneumonia as we performed the rapid-sequence anesthetic induction, and the tracheal aspiration after intubation had not showed a stigma of gastric liquid inhalation. Acute hemolytic transfusion reaction was ruled out by the absence of hemoglobinuria and falling hemoglobin levels.

The hypoxemia worsened despite protective ventilation adapted to acute respiratory distress syndrome (ARDS). Subsequently, the mean arterial pressure decreased to $50 \mathrm{mmHg}$, requiring an increasing dose of noradrenaline and the introduction of dobutamine. In this context of severe hypoxemia, the patient progressed to multiple organ failure and died the day after the surgery.

\section{Discussion}

TRALI is defined as new acute lung injury (ALI) that develops during or within 6 hours of transfusion of one or more units of blood products, not attributable to another ALI risk factor (Table 1). ${ }^{3,4}$ Patients who concurrently have

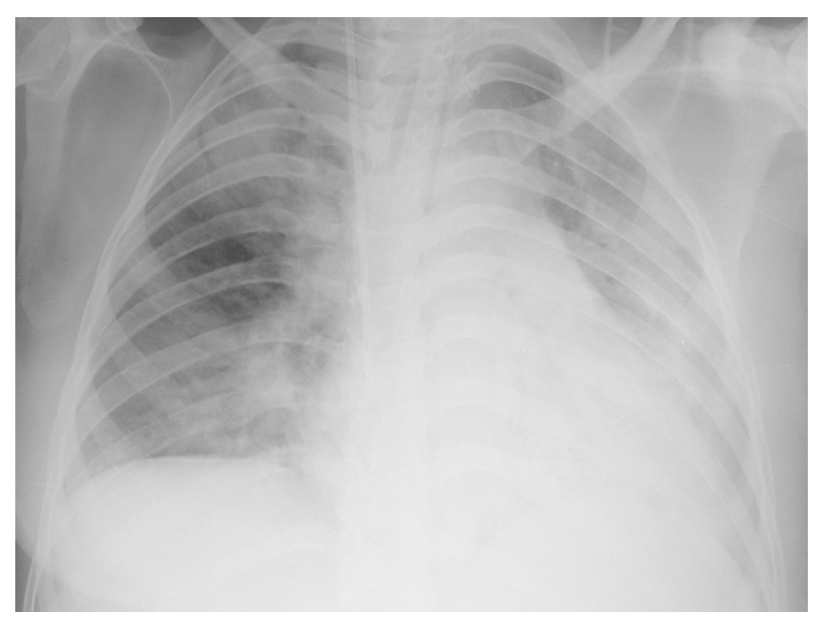

Figure I Chest radiography after the transfusion, revealing newly developed diffuse bilateral lung infiltrations in mostly both whole lungs. 
Table I Definition of transfusion-related acute lung injury

\begin{tabular}{ll}
\hline Suspected & - Acute onset within $6 \mathrm{~h}$ of the end of blood \\
TRALI & transfusion \\
& - $\mathrm{PaO}_{2} / \mathrm{FiO}_{2}<300 \mathrm{mmHg}$ \\
& - Pilateral infiltrates on frontal chest radiographs \\
& Pulmonary capillary wedge pressure of $18 \mathrm{mmHg}$ \\
& hypertension
\end{tabular}

Note: Data from Goldman et al ${ }^{3}$ and Bernard et al. ${ }^{4}$

Abbreviations: h, hours; $\mathrm{FiO}_{2}$, fraction of inspired oxygen; $\mathrm{PaO}_{2}$, partial pressure of arterial oxygen; TRALI, transfusion-related acute lung injury.

another major ALI risk factor (pneumonia, sepsis, aspiration, multiple fractures, or pancreatitis) are excluded from the diagnosis and labeled with "possible TRALI". These newly established diagnostic criteria have increased the awareness of TRALI and resulted in a recent increase in reports of this syndrome. Consequently, TRALI has emerged now as the most important cause of morbidity and mortality resulting from blood transfusion. . $^{5-7}$

All plasma-containing blood and blood components have been implicated (whole blood, packed cells, FFP, platelet concentrates produced from whole blood donation or collected by apheresis, granulocytes, and, rarely, intravenous immunoglobulin and cryoprecipitate). ${ }^{6}$ Incidence rates vary widely because of the lack of large prospective studies using a standard definition and also, because of the variability of the studied population; however, the incidence ranges from $0.08 \%$ per inpatient transfused ${ }^{8}$ to $8 \%$ in critically ill patients. ${ }^{9}$ Estimates of the incidence of TRALI are one in 5,000 units of transfused blood product, ${ }^{10}$ one in 7,900 units of FFP, ${ }^{11}$ and one in 432 units of whole blood-derived platelet concentrates. ${ }^{9}$

The pathogenesis of TRALI is still debated. Classically, TRALI was considered as the consequence of an immunologically mediated reaction, occurring through the binding of donor antibodies against recipient leukocyte antigens, with the antibodies of the donor being passively infused during the transfusion of the blood components. ${ }^{12}$ These antibodies are directed against specific human neutrophil antigens or human leukocyte antigens in the lungs of the recipient. A two-event model has been proposed for TRALI: ${ }^{13}$ first is the occurrence of underlying patient factors, such as an inflammatory condition, causing an adherence of primed neutrophils to the pulmonary endothelium; the second event is caused by the transfusion of blood products containing either antibodies or bioactive lipids that have accumulated during blood storage, stimulating the primed neutrophils to release proteases. The result is endothelial damage, capillary leak, extravasation of neutrophils, and subsequent pulmonary edema. ${ }^{14,15}$ The antibody-mediated TRALI investigation strategy must include the detection of antibodies to human neutrophil alloantigen (HNA), human leukocyte antigen (HLA)-A2, and HLA class II. ${ }^{16}$ Over $20 \%$ of TRALI events have no associated leukocyte antibodies, consistent with the hypothesis that biological factors other than antibodies may precipitate TRALI. ${ }^{16}$ Other data have indicated that aged blood products have biological response modifiers that contribute to TRALI pathogenesis. ${ }^{16}$ In our case, the age of the transfused PRBC and FFP was acceptable and was, respectively, 8 and 14 days; however, we did not perform an antibody-mediated TRALI investigation, due to the absence of adequate laboratory facilities in our institution.

Respiratory disorders, including dyspnea, tachypnea, and hypoxemia, are the central clinical symptoms in TRALI. Such problems are a result of increased pulmonary vascular permeability and ensuing lung edema. However, a wide range of other reactions can take place because of antibody infusion, including rigors, tachycardia, fever, hypothermia, hypotension, and, rarely, hypertension. ${ }^{17}$

In mechanically ventilated patients, the diagnosis should be considered whenever there is an acute, unexplained worsening in respiratory status that is temporally associated with a transfusion. In such patients, copious frothy pink edema fluid is often recovered from the endotracheal tube. ${ }^{18}$ There is no specific laboratory testing to confirm TRALI - the only parameter that has been associated with TRALI is leukopenia, especially neutropenia, ${ }^{19}$ which arises only in $5 \%-35 \%$ of patients and was absent in our case. Neutropenia is thought to be due to neutrophil-specific antibodies. ${ }^{20}$

Although the diagnosis of TRALI is primarily clinical and radiographic, it is important to differentiate TRALI clinically from transfusion-associated cardiac overload (TACO) and other transfusion reactions, like allergic reactions and bacterial contamination of the transfused blood product. The clinical profile of the patient at risk for TACO is 3 years or younger, or older than 60 . Respiratory distress and/or cyanosis associated with pulmonary edema presents within 2 hours of transfusion. Elevated blood pressure, tachycardia, and increased pulmonary wedge pressure are the typical stigmata. ${ }^{21}$ Elevated CVP or pulmonary artery occlusion pressure, pulmonary edema with cardiomegaly on chest radiography, and prompt response to diuretics are more consistent with TACO. The CVP and wedge pressures are typically low or normal in TRALI but may be elevated if 
TRALI occurs in the setting of heart failure. ${ }^{19}$ In our case, the echocardiography was normal, and the CVP was at $5 \mathrm{cmH}_{2} \mathrm{O}$, within normal range; those elements favored permeability pulmonary edema. A septic transfusion reaction can present as TRALI; if signs of sepsis are present, sepsis treatment should be started promptly, even while Gram stain and culture of the blood bag and blood cultures of the patient are pending. Anaphylactic transfusion reactions or toxic shock syndrome, including tachypnea and wheezing, also present with respiratory distress. Because symptoms are a result of laryngeal and bronchial edema and not of pulmonary edema, a chest radiograph can aid diagnosis. ${ }^{18}$ Since there were no symptoms of allergic reaction, like urticaria, erythema of the face and trunk, or wheezing, the diagnosis of anaphylactic transfusion reaction was also excluded. Acute hemolytic reaction was excluded based on biological evidence and the absence of typical clinical features, such as hemoglobinuria or renal failure.

Amniotic fluid embolism was another possible differential diagnosis of our case. This is a rare obstetric condition, characterized by sudden cardiovascular collapse, altered mental status, and disseminated intravascular coagulation. The collapse often occurs during labor or in the immediate postpartum period. The current available evidence suggests that the hemodynamic response to amniotic fluid embolism is biphasic, with initial increased pulmonary vascular resistance and right ventricular failure, followed by left ventricular failure. ${ }^{22}$ In our case, the echocardiography showed no abnormalities in the right or left heart function; in addition, there was no disseminated intravascular coagulation or acute fetal distress. The acute respiratory failure occurred late in the cesarean section and after the transfusion of blood products.

Our patient had clinical evidence of acute hypoxemia 3 hours after the end of the transfusion. The $\mathrm{PaO}_{2} / \mathrm{FiO}_{2}$ was 51 , and lung auscultation revealed diffuse crackles. The chest radiography was characteristic of pulmonary edema, and we did not find any other ALI/ARDS risk factor except transfusion. Therefore, our case corresponds to all criteria of definition of TRALI (Table 1). However, we noted a rapid progression to multiple organ failure. We believe that continuous and deep hypoxemia, with a $\mathrm{PaO}_{2}$ below $50 \mathrm{mmHg}$ despite adapted ventilation, was the main cause. This severe hypoxemia could have been improved by establishing therapeutics used in ALI or ARDS, including nitric oxide inhalation or extracorporeal membrane oxygenation. Unfortunately, those therapeutics were not available in our institution, and the hypoxemia continued to worsen, favoring the progression to multiple organ failure.
As with other forms of ALI, there is no specific treatment for TRALI. In mild TRALI cases, clinical improvement is generally achieved only with nasal oxygen administration. However, $70 \%$ of the patients presenting the most severe form require orotracheal intubation and mechanical ventilation. ${ }^{23}$ Restrictive tidal volume ventilation should be employed, as would be used in cases of ARDS. ${ }^{24}$ For severe cases, mechanical ventilation, intravenous fluids, invasive hemodynamic monitoring, and vasopressors may be required. In rare cases, the hypoxemia resulting from TRALI can be so severe that extracorporeal membrane oxygenation may be required as a temporizing measure while the lungs heal. ${ }^{25,26}$ Potentially harmful interventions, such as the administration of diuretics, should be avoided. In fact, patients with TRALI who are hypotensive may require intravenous fluids to maintain an adequate blood pressure. ${ }^{18}$ Nevertheless, the hypotension may be unresponsive to intravenous fluids in the presence of normal cardiac function, as was seen in our patient, who was then in need of vasopressor support. As in patients with ARDS, corticosteroids, nonsteroidal antiinflammatory drugs, and surfactant protein, as well as other therapeutic agents, do not provide benefits for patients with TRALI. ${ }^{12,27}$ As TRALI is underdiagnosed and underreported in Morocco, there are no specific strategies to prevent it; in fact, we still produce FFP units from woman donors and we don't achieve any detection of donor leukocyte specific antibodies. We believe that some measures could be taken to limit the risk of TRALI in this setting in the future. Certainly, avoiding unnecessary blood transfusions will reduce the incidence of TRALI. Donors who have antibodies to high-frequency leukocyte antigens should be disqualified from plasma or platelet donation. Also, use of only male donors to manufacture FFP, use of pooled plasma preparations instead of single-donor plasma, decreasing the amount of plasma remaining in red cell components, and the use of fresher products are some of the methods that should be undertaken to prevent this condition. ${ }^{28}$

\section{Conclusion}

TRALI is a serious complication of transfusion. The incidence is high, especially in at-risk patient populations, such as the critically ill. Both the underlying condition of the patient and transfusion factors play a role in the onset of this pathology. TRALI also needs to be distinguished from TACO, anaphylactic transfusion reactions, and bacterial contamination of the transfused blood product.

TRALI is a clinical diagnosis that should be considered in all patients who have pulmonary insufficiency during or 
after transfusion. Timely recognition and adequate treatment is crucial in its management. ${ }^{28}$

\section{Disclosure}

The authors report no conflicts of interest in this work.

\section{References}

1. Looney MR, Gropper MA, Matthay MA. Transfusion-related acute lung injury. A review. Chest. 2004;126(1):249-258.

2. US Food and Drug Administration. Fatalities Reported to FDA Following Blood Collection and Transfusion: Annual Summary for Fiscal Year 2009. Rockville, MD: US Department of Health and Human Services; 2010.

3. Goldman M, Webert KE,ArnoldDM, Freedman J, Hannon J, Blajchman MA. Proceedings of a consensus conference: towards an understanding of TRALI. Transfus Med Rev. 2005;19:2-31

4. Bernard GR, Artigas A, Brigham KL, et al. The American-European Consensus Conference on ARDS. Definitions, mechanisms, relevant outcomes, and clinical trial coordination. Am J Respir Crit Care Med. 1994;149:818-824.

5. Holness L, Knippen MA, Simmons L, Lachenbruch PA. Fatalities caused by TRALI. Transfus Med Rev. 2004;18(3):184-188.

6. Toy P, PopovskyMA, Abraham E, et al; National Heart, Lung and Blood Institute Working Group on TRALI. Transfusion-related acute lung injury: definition and review. Crit Care Med. 2005;33(4):721-726.

7. Kleinman S, Caulfield T, Chan P, et al. Toward an understanding of transfusion-related acute lung injury: statement of a consensus panel. Transfusion. 2004;44(12):1774-1789.

8. Gajic O, Rana R, Winters JL, et al. Transfusion-related acute lung injury in the critically ill: prospective nested case-control study. Am J Respir Crit Care Med. 2007;176(9):886-891.

9. Silliman CC, Boshkov LK, Mehdizadehkashi Z, et al. Transfusionrelated acute lung injury: epidemiology and a prospective analysis of etiologic factors. Blood. 2003;101(2):454-462.

10. Popovsky MA, Moore SB. Diagnostic and pathogenetic considerations in transfusion-related acute lung injury. Transfusion. 1985;25(6):573-577.

11. Wallis JP, Lubenko A, Wells AW, Chapman CE. Single hospital experience of TRALI. Transfusion. 2003;43(8):1053-1059.

12. Silliman CC, Ambruso DR, Boshkov LK. Transfusion-related acute lung injury. Blood. 2005;105(6):2266-2273

13. Marik PE, Corwin HL. Acute lung injury following blood transfusion: expanding the definition. Crit Care Med. 2008;36(11):3080-3084.
14. Looney MR, Su X, Van Ziffle JA, Lowell CA, Matthay MA. Neutrophils and their Fc gamma receptors are essential in a mouse model of transfusion-related acute lung injury. $J$ Clin Invest. 2006;116(6):1615-1623.

15. Silliman CC, Bjornsen AJ, Wyman TH, et al. Plasma and lipids from stored platelets cause acute lung injury in an animal model. Transfusion. 2003;43(5):633-640.

16. Fung YL. Transfusion-related acute lung injury investigation insights. ISBT Science Series. 2011;6(1):206-211.

17. Vlaar AP, Juffermans NP. Transfusion-related acute lung injury: a clinical review. Lancet. 2013;382(9896):984-994.

18. Sayah DM, Looney MR, Toy P. Transfusion reactions: newer concepts on the pathophysiology, incidence, treatment, and prevention of transfusion-related acute lung injury. Crit Care Clin. 2012;28(3):363-372, v.

19. Borazan H, Yosunkaya S, Yosunkaya A. Transfusion related acute lung injury: A severe case triggered with anti-HLA class II antibodies in the recipient. Signa Vitae. 2012;7(1):52-56.

20. Fadeyi EA, De Los Angeles Muniz M, Wayne AS, Klein HG, Leitman SF, Stroncek DF. The transfusion of neutrophil-specific antibodies causes leukopenia and a broad spectrum of pulmonary reactions. Transfusion. 2007;47(3):545-550.

21. Popovsky MA. Pulmonary consequences of transfusion: TRALI and TACO. Transfus Apher Sci. 2006;34(3):243-244.

22. Conde-Agudelo A, Romero R. Amniotic fluid embolism: an evidencebased review. Am J Obstet Gynecol. 2009;201(5):445.e1-445.e13.

23. Fabron A Jr, Lopes LB, Bordin JO. Transfusion-related acute lung injury. J Bras Pneumol. 2007;33(2):206-212.

24. Ventilation with lower tidal volumes as compared with traditional tidal volumes for acute lung injury and the acute respiratory distress syndrome. The Acute Respiratory Distress Syndrome Network. NEngl J Med. 2000;342(18):1301-1308.

25. Lee AJ, Koyyalamudi PL, Martinez-Ruiz R. Severe transfusion-related acute lung injury managed with extracorporeal membrane oxygenation (ECMO) in an obstetric patient. J Clin Anesth. 2008;20(7): $549-552$.

26. Kuroda H, Masuda Y, Imaizumi H, Kozuka Y, Asai Y, Namiki A. Successful extracorporeal membranous oxygenation for a patient with life-threatening transfusion-related acute lung injury. $J$ Anesth. 2009;23(3):424-426.

27. Webert KE, Blajchman MA. Transfusion-related acute lung injury. Curr Opin Hematol. 2005;12(6):480-487.

28. Aravinthan A, Sen S, Marcus N. Transfusion-related acute lung injury: a rare and life-threatening complication of a common procedure. Clin Med. 2009;9(1):87-89.
International Journal of Clinical Transfusion Medicine

\section{Publish your work in this journal}

International Journal of Clinical Transfusion Medicine is an international, peer-reviewed, open access, online journal publishing clinicalexperimental, policy-making and evidence-based practices of all topics pertaining to clinical transfusion medicine. Original research, short reports, reviews, case reports and commentaries are invited.

\section{Dovepress}

The manuscript management system is completely online and includes a very quick and fair peer-review system, which is all easy to use. Visit http://www.dovepress.com/testimonials.php to read real quotes from published authors. 